\title{
ESTUDO DA TEMPERATURA DE FUSÃO E SOLUBILIDADE DOS CRISTAIS DE LACTOSE EM LEITE CONDENSADO UTILIZANDO MICROSCOPIA ÓPTICA E ESPECTROSCOPIA RAMAN
}

\author{
Study of the melting point and solubility of lactose crystals in \\ sweetened condensed milk using optical microscopy and Raman \\ spectroscopy
}

Natália Maria Germano Alves ${ }^{1}$, Elisângela Ramieres Gomes ${ }^{2}$, João Pablo Fortes Pereira ${ }^{3}$, Ítalo Tuler Perrone ${ }^{4}$, Luiz Fernando Cappa de Oliveira ${ }^{1}$, Rodrigo Stephani ${ }^{1 *}$

\section{RESUMO}

O estudo dos parâmetros físico-químicos como a temperatura de fusão e a solubilidade dos cristais de lactose no leite condensado justifica-se pela necessidade de solubilização de cristais de lactose em algumas etapas do processamento deste produto nas indústrias bem como a ausência destes dados na literatura científica. A presença de grandes cristais de lactose no leite condensado é indesejável ao consumidor final, comprometendo assim a qualidade sensorial do produto. $\mathrm{O}$ objetivo deste trabalho foi determinar a temperatura de fusão dos cristais de lactose e a proporção ajustada da solução de reprocessamento, composta da mistura de leite condensado/diluente, fornecendo duas metodologias alternativas para aperfeiçoar a qualidade do produto. Utilizou-se um sistema controlador de temperatura acoplado ao microscópio óptico para acompanhar a rampa de aquecimento até a fusão efetiva dos cristais de lactose nas amostras de leite condensado, soluções de reprocessamento e na solução diluente, onde obteve-se a temperatura média de fusão dos cristais no leite condensado que foi de $83,3^{\circ} \mathrm{C}$. Utilizou-se a análise microscópica nas amostras e a partir das micrografias das soluções de reprocessamento, pode concluir que a amostra $\mathrm{T}_{20}$ seria a mais adequada para corrigir o defeito de arenosidade. A partir do

1 Universidade Federal de Juiz de Fora, Departamento de Química, Rua José Lourenço Kelmer, s/n, São Pedro, 36036-900, Juiz de Fora, MG, Brasil.E-mail:rodrigostephani@gmail.com

2 Universidade Federal de Viçosa, Departamento de Tecnologia de Alimentos, Viçosa, MG, Brasil.

3 Universidade Federal de Juiz de Fora, Departamento de Nutrição, Juiz de Fora, MG, Brasil.

4 Universidade Federal de Juiz de Fora, Faculdade de Farmácia, Juiz de Fora, MG, Brasil.

* Autor para correspondência

Recebido / Re ceived: 23/03/2020

Aprovado / Approved: 16/10/2020 
espectro Raman observou-se o perfil espectroscópico de todas as amostras e atribuiuse às bandas características de cristalização dos açúcares. Desta forma, foi possível determinar os parâmetros de temperatura e diluição para o reprocessamento do leite condensado visando minimizar o problema da arenosidade.

Palavras-chave: açúcares; arenosidade; reprocesso; diluente.

\begin{abstract}
The study of physical-chemical parameters such as the melting point and lactose crystals solubility in sweetened condensed milk is justified by process stage demand of this product in industries, as well as the lack of these data in the scientific literature. The undesirable presence of large lactose crystals in sweetened condensed milk compromises the sensory quality of the final product. The aim of this study was to determine the melting point of the lactose crystals and the adjusted proportion of the reprocessing solution, made up of a mixture of sweetened condensed milk/diluent. The study provided two alternative methodologies to enhance the quality of the final product. A temperature control system coupled to the optical microscope was used to monitor the heating ramp until the effective melting of the lactose crystals in the samples of sweetened condensed milk, reprocessing solutions, and diluent solution. The average melting temperature of crystals in sweetened condensed milk was 83.3 ${ }^{\circ} \mathrm{C}$. Microscopic analyzes were performed on the samples, and from micrographs of the reprocessing solutions, it was concluded that the $\mathrm{T}_{20}$ sample is the most suitable to correct the problem of sandiness. The spectroscopic profile of all samples was observed by Raman spectroscopy and attributed to the characteristic bands of sugar crystallization. Therefore, it was possible to determine the temperature and dilution parameters for the reprocessing of sweetened condensed milk in order to minimize the problem of sandiness.
\end{abstract}

Keywords: sugars; sandiness; reprocessing; diluent.

\section{INTRODUÇÃO}

Leite condensado é o produto resultante da desidratação parcial do leite, leite concentrado ou leite reconstituído, com adição de açúcar, podendo ter seus teores de gordura e proteína ajustados unicamente para o atendimento das características do produto (BRASIL, 2018a). Pela definição química, consiste predominantemente de uma solução saturada de lactose. De acordo com o Codex Alimentarius (2006), o leite condensado possui composição mínima de: $8 \% \mathrm{~m} / \mathrm{m}$ de gordura láctea, $28 \% \mathrm{~m} / \mathrm{m}$ de sólidos lácteos e $34 \% \mathrm{~m} / \mathrm{m}$ de proteína láctea nos sólidos lácteos não gordurosos. Pode ser obtido pela remoção parcial da água do leite e posterior adição de sacarose ou por qualquer outro processo que conduza a um produto com a mesma composição e características (RENHE et al., 2011).

A cristalização da lactose no leite condensado após a evaporação e a adição de sacarose é induzida durante o resfriamento do produto, e ocorre devido à alta concentração de lactose ( $>37 \mathrm{~g}$ de lactose/100 g de água), além de uma elevada concentração de sacarose ( $>157 \mathrm{~g}$ de sacarose/100 g de água) (PEREIRA et al., 2019).

A formação dos cristais de lactose 
no leite condensado por meio da indução denomina-se nucleação secundária e tem como finalidade a obtenção de um produto final com melhores atributos sensoriais (NICKERSON, 1974). A cristalização deve ocorrer pela inserção de microcristais de lactose e em seguida feito o resfriamento, em condições ajustadas, para que se possa evitar à formação de grandes cristais (PERRONE et al., 2019). A nucleação secundária deve ocorrer antes da cristalização espontânea da lactose, seguido pelo controle de temperatura e taxa de agitação, com a finalidade de se obter um produto de textura suave e homogênea (SIMEÃO, 2018).

A solubilidade pode ser definida pela quantidade máxima de uma dada substância, em gramas, que pode ser solubilizada em $100 \mathrm{~g}$ de água, sendo função da temperatura. A solubilidade da lactose no leite condensado é diminuída com o aumento de sacarose no meio, e também diminuída pela elevação da temperatura do sistema, sendo este efeito mais evidente em soluções com alta concentração (NICKERSON; MOORE, 1972). A falta de controle de tais parâmetros pode ocasionar defeitos no produto, em especial a arenosidade, no qual os cristais de lactose são perceptíveis ao paladar, possuindo tamanhos superiores a $25 \mu \mathrm{m}$ (SILVA et al., 2015; STEPHANI et al., 2017). Portanto, faz-se necessário, em alguns casos, à inclusão da etapa de reprocessamento na linha de produção afim de obter a qualidade desejada do produto final.

A arenosidade pode ser determinada com o auxílio da técnica de microscopia óptica, na qual pode-se observar a presença de cristais no produto a olho nu. Outra técnica alternativa para a determinação de cristais é a espectroscopia Raman.

A microscopia óptica é uma das técnicas experimentais mais exploradas para análise de cristais em amostras de alimentos, sendo possível com a utilização da mesma avaliar o tamanho, o número de cristais, a taxa de cristalização e os tipos de cristais presentes em uma determinada amostra (SCHUMACHER, 2015).

A espectroscopia Raman é uma técnica muito vantajosa e rápida, em que a amostra não necessita de tratamento prévio. Os resultados são obtidos por espectros característicos de cada modo vibracional presente na amostra, que podem-se relacionar com as bandas características de cristalização de um dado componente (ALMEIDA, 2011). O microscópio óptico do espectrômetro Raman acoplado a um controlador de temperatura, possibilita a análise de inúmeros parâmetros físico-químicos visando melhor entendimento do controle de qualidade de alimentos.

Neste contexto, o objetivo desse trabalho foi determinar a temperatura de fusão completa dos cristais de lactose presentes na amostra de leite condensado, além de obter a proporção ajustada da diluição das amostras de leite condensado em uma solução de sacarose (diluente) propiciando a solubilização completa dos cristais.

\section{MATERIAL E MÉTODOS}

\section{Amostras}

As amostras de leite condensado bem com o leite pasteurizado integral foram obtidas de um mesmo lote de uma empresa localizada no estado de Minas Gerais.

Foram preparadas soluções de reprocessamento $\left(\mathrm{T}_{90}, \mathrm{~T}_{80}, \mathrm{~T}_{50}\right.$ e $\left.\mathrm{T}_{20}\right)$ com proporções pré-definidas baseando-se em valores utilizados atualmente pela indústria alimentícia. As soluções de reprocessamento consistem na mistura de leite condensado e diluente, sendo este composto por sacarose e leite pasteurizado (na proporção $20 \mathrm{~g}$ de sacarose em $100 \mathrm{~g}$ de leite pasteurizado). As amostras e suas respectivas proporções são apresentadas na Tabela 1 . Todas as soluções foram preparadas 24 horas antes das análises. 


\section{Microscopia}

Na literatura não há uma metodologia específica para a análise dos cristais de lactose em produtos lácteos. Desse modo, adaptou-se a metodologia utilizada no estudo de Hough et al. (1990) para a avaliação microscópica dos cristais de lactose em doce de leite. Utilizou-se o microscópio óptico trinocular monocromático da marca Medlux, modelo MC30, com área do campo microscópico de $\sim 0,1 \mathrm{~mm}^{2}$, utilizando a lente objetiva de $40 \mathrm{x}$ e abertura numérica de 0,85 .

Foram preparadas seis lâminas de cada uma das amostras descritas na Tabela 1 para avaliação do perfil, tamanho e contagem dos cristais. Pesou-se exatamente cerca de $3 \mathrm{mg}$ a $4 \mathrm{mg}$ de cada uma das amostras sobre a lâmina, em seguida, mediu-se o diâmetro das mesmas, sendo este relacionado ao tamanho médio dos cristais. A partir de cada lâmina obteve-se 10 micrografias correspondendo a 10 campos aleatórios (RENHE et al., 2018). As análises foram realizadas na temperatura ambiente $\left(\sim 25^{\circ} \mathrm{C}\right)$, sem tratamento prévio das amostras.

A contagem média do número de cristais de lactose por grama em cada campo foi realizada mediante a utilização da Equação 1 (HOUGH et al., 1990):

$$
N=\frac{n F \cdot \pi \cdot R^{2}}{F \cdot W}
$$

Em que:

$N=$ número de cristais por grama de leite condensado;

$n F=$ média do número de cristais observados (10 campos aleatórios);

$R^{2}=$ raio do círculo formado pela amostra entre a lâmina e a lamínula elevado ao quadrado $\left(\mathrm{mm}^{2}\right)$;

$F=$ área do campo microscópico $\left(\mathrm{mm}^{2}\right)$;

$W=$ massa da amostra de leite condensado $(\mathrm{g})$.

\section{Análises físico-químicas}

As análises do leite pasteurizado e da amostra de leite condensado realizadas foram: umidade pelo método gravimétrico (IDF, 1988), gordura pelo método de Gerber (IDF, 2008), teor de proteína pelo método de Kjeldahl (IDF, 1993), resíduo mineral fixo pelo método gravimétrico (CUNNIFF, 1997), lactose pelo método de Lane \& Eynon (BRASIL, 2006), sólidos totais obtido pela diferença entre o valor da constituição total e a umidade, sólidos solúveis por refratometria, ${ }^{\circ}$ Brix, e a sacarose obtida por diferença (RENHE et al., 2011).

\section{Temperatura de fusão dos cristais de lactose}

Para determinação da temperatura de fusão dos cristais de lactose das amostras, utilizou-se o microscópio BX51 com fonte de

Tabela 1 - Descrição da composição de todas as amostras analisadas

\begin{tabular}{cc}
\hline Identificação & Composição $(\% \mathbf{~ m} / \mathbf{m})$ \\
\hline LC & Leite condensado \\
Diluente & 20g de sacarose $+100 \mathrm{~g}$ de leite pasteurizado \\
T90 & $90 \mathrm{~g}$ de leite condensado $+10 \mathrm{~g}$ do diluente $(90 / 10)$ \\
T80 & 80g de leite condensado $+20 \mathrm{~g}$ do diluente $(80 / 20)$ \\
T50 & $50 \mathrm{~g}$ de leite condensado $+50 \mathrm{~g}$ do diluente $(50 / 50)$ \\
T20 & 20g de leite condensado $+80 \mathrm{~g}$ do diluente $(20 / 80)$ \\
\hline
\end{tabular}


iluminação alógena brilhante $12 \mathrm{~V} / 100 \mathrm{~W}$ com lente objetiva de 50x, acoplado a um sistema de controle de temperatura modelo Linkan FTIR 600 no qual possui platina termométrica para infravermelho $\left(-196{ }^{\circ} \mathrm{C}\right.$ até $\left.600{ }^{\circ} \mathrm{C}\right)$. Utilizou-se uma rampa de aquecimento com uma faixa de temperatura entre $25^{\circ} \mathrm{C}$ e $70^{\circ} \mathrm{C}$ para cada amostra $(n=3)$, e as micrografias foram obtidas de $5{ }^{\circ} \mathrm{C}$ em $5{ }^{\circ} \mathrm{C}$. A taxa de aquecimento durante todas as análises foi de $3{ }^{\circ} \mathrm{C} / \mathrm{min}$. A observação da fusão completa dos cristais foi realizada de forma direta no microscópio e registrada por gravação de vídeos.

\section{Espectroscopia Raman}

Para a obtenção dos espectros Raman e posterior avaliação do efeito da solubilização dos cristais de lactose das amostras, utilizou-se o espectrômetro Bruker FT- Raman RFS 100 equipado com detector de GE refrigerado com nitrogênio líquido, e emissão de laser Nd: YAG com excitação em $1064 \mathrm{~nm}$. As amostras foram colocadas em cubetas de quartzo 10x10 mm com incidência do laser com potência de 200 $\mathrm{mW}$. Realizou-se 512 scans, os quais foram coletados com resolução espectral de $4 \mathrm{~cm}^{-1}$ em uma região compreendida entre $3200 \mathrm{~cm}^{-1}$ e $200 \mathrm{~cm}^{-1}$ (ALMEIDA, 2011).

\section{RESULTADOS E DISCUSSÃO}

Os resultados obtidos das análises físico-químicas do leite pasteurizado e do leite condensado estão apresentados na Tabela 2 .

A composição do leite pasteurizado e do leite condensando estão de acordo com os padrões exigidos pelo Ministério da Agricultura Pecuária e Abastecimento (MAPA) e Codex Alimentarius (BRASIL, 2018b; RENHE et al., 2011).

Na Figura 1 estão apresentadas as micrografias referentes a amostra de leite condensado (LC), das soluções de reprocessamento $\left(\mathrm{T}_{90}, \mathrm{~T}_{80}, \mathrm{~T}_{50}\right.$ e $\left.\mathrm{T}_{20}\right)$ assim como as micrografias da solução diluente. Observa-se que quanto maior a quantidade da solução diluente, menor a quantidade de cristais de lactose inicialmente presentes no meio nas temperaturas entre $25{ }^{\circ} \mathrm{C}$ e $30{ }^{\circ} \mathrm{C}$. O leite condensado é um produto no qual quanto menor o tamanho dos cristais, melhor será a qualidade do produto final.

A quantidades de cristais, bem como as médias dos seus respectivos tamanhos

Tabela 2 - Composição físico-química do leite pasteurizado e do leite condensado

\begin{tabular}{ccc}
\hline Parâmetros & Leite pasteurizado & Leite condensado \\
\hline Sólidos totais (\%) & $12,1 \pm 0,1$ & $73,3 \pm 0,6$ \\
Gordura (\%) & $3,4 \pm 0,1$ & $8,2 \pm 0,2$ \\
Proteína (\%) & $2,9 \pm 0,1$ & $6,3 \pm 0,2$ \\
Lactose (\%) & $4,7 \pm 0,1$ & $10,8 \pm 0,7$ \\
Resíduo Mineral fixo (\%) & $0,9 \pm 0,1$ & $1,4 \pm 0,2$ \\
Sacarose (\%) & $*$ & $45,7 \pm 0,6$ \\
Sólidos Solúveis $\left({ }^{\circ}\right.$ Brix) & $*$ & $71,0 \pm 0,5$ \\
\hline
\end{tabular}

*não analisado 
nas amostras estão apresentadas na Tabela 3. Esses resultados corroboram aos resultados obtidos através das micrografias. Observase a partir das micrografias da solução de reprocessamento $T_{20}$ que não há no sistema lactose na forma cristalina (cristal do tipo tomahawk), foi encontrado somente algumas estruturas globulares que não se referem à lactose. Portanto, a solução $\mathrm{T}_{20}$ teria a proporção mais aplicável para a indústria de leite condensado.

As micrografias referentes a rampa de aquecimento, visando identificação da temperatura de fusão dos cristais de lactose para cada amostra também estão representadas na Figura 1. É possível perceber a influência da concentração do diluente na temperatura de fusão dos cristais presentes nas soluções de
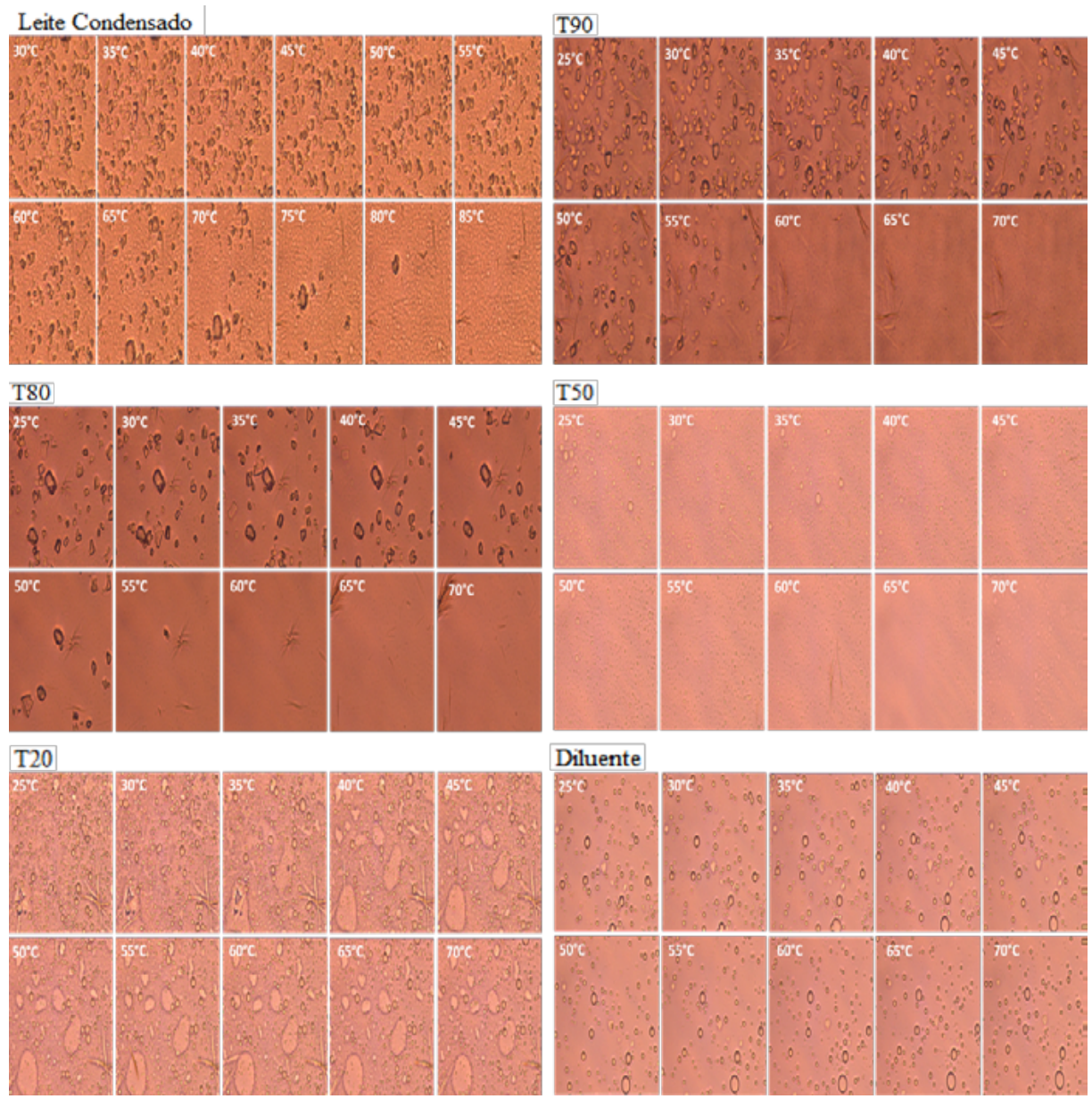

Figura 1 - Micrografias com aumento de 500x obtidas em diferentes temperaturas: LC (leite condensado); $\mathrm{T}_{90}\left(90 \mathrm{~g}\right.$ de $\mathrm{LC}$ e $10 \mathrm{~g}$ de diluente); $\mathrm{T}_{80}\left(80 \mathrm{~g}\right.$ de LC e $20 \mathrm{~g}$ de diluente); $\mathrm{T}_{50}(50$ $\mathrm{g}$ de LC e $50 \mathrm{~g}$ de diluente); $\mathrm{T}_{20}(20 \mathrm{~g}$ de LC e $80 \mathrm{~g}$ de diluente); Diluente (solução de $20 \mathrm{~g}$ de sacarose em $100 \mathrm{~g}$ de leite pasteurizado). 
reprocessamento $\mathrm{T}_{90}, \mathrm{~T}_{80}$ e $\mathrm{T}_{50}$, assim como a influência do aquecimento nos cristais de lactose presentes no leite condensado. Podemos observar que há uma diminuição da temperatura de fusão dos cristais de lactose nas amostras com maior concentração do diluente, pois com o aumento da diluição há uma menor saturação da lactose consequentemente menor quantidade de cristais presentes em solução, conforme os dados apresentados na Tabela 3. As concentrações teóricas de lactose nas amostras estudadas estão apresentadas na Tabela 4, essas foram obtidas utilizando os dados de composição físico-química dos produtos.

Não há relatos na literatura de dados sobre a relação da temperatura na solubilidade da lactose aplicado na amostra de leite condensado. Objetivando uma projeção teórica, utilizou-se uma solução modelo, ou seja, uma solução de lactose em água. Assim, a partir dos dados obtidos da temperatura de fusão experimental de cada amostra e da solubilidade inicial da $\alpha$-lactose apresentados por Whittier (1944) e Schuck (2018), foi possível estimar a solubilidade da lactose em leite condensado (Tabela 4).

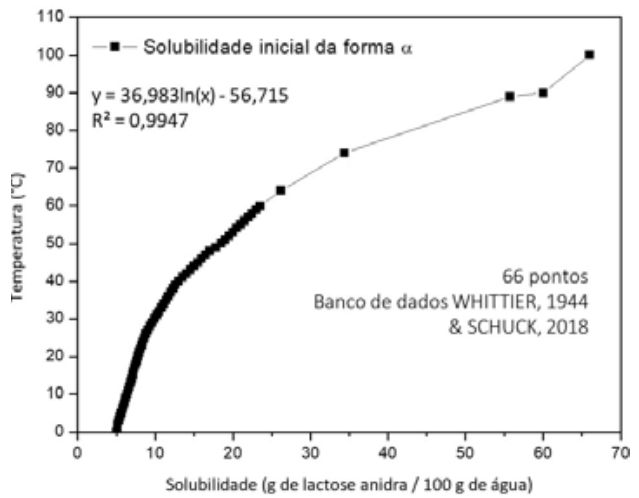

Figura 2 - Curva de solubilidade inicial da $\alpha$-lactose em água, construída utilizando os dados combinados de Whittier (1944) e Schuck (2018)

Comparando-se os valores de temperatura de fusão experimental com os propostos do modelo de Whittier e Shuck, pode-se concluir que os mesmos são bem próximos, mas não se encontram dentro do intervalo de confiança. Isso se deve ao fato de que os trabalhos encontrados na literatura científica se baseiam na solubilidade da

Tabela 3 - Quantidade de cristais de lactose por grama de leite condensado e tamanho médio dos cristais

\begin{tabular}{ccc}
\hline \multirow{2}{*}{ Amostra $^{*}$} & \multicolumn{2}{c}{ Cristais de Lactose } \\
\cline { 2 - 3 } & Quantidade $\left(\mathbf{n}^{\mathbf{0}} / \mathbf{g}\right)$ & Tamanho $(\boldsymbol{\mu m})$ \\
\hline $\mathrm{LC}$ & $(9,3 \pm 1,0) \times 10^{8}$ & $9,8 \pm 1,0$ \\
$\mathrm{~T}_{90}$ & $(4,0 \pm 1,0) \times 10^{8}$ & $18,7 \pm 1,0$ \\
$\mathrm{~T}_{80}$ & $(3,5 \pm 1,0) \times 10^{8}$ & $18,8 \pm 1,0$ \\
$\mathrm{~T}_{50}$ & $(1,9 \pm 1,0) \times 10^{7}$ & $14,2 \pm 2,0$ \\
$\mathrm{~T}_{20}$ & $\mathrm{X}$ & $\mathrm{X}$ \\
Diluente & $\mathrm{X}$ & $\mathrm{X}$ \\
\hline
\end{tabular}

* LC (leite condensado); $\mathrm{T}_{90}$ (90 $\mathrm{g}$ de $\mathrm{LC}$ e $10 \mathrm{~g}$ de diluente); $\mathrm{T}_{80}\left(80 \mathrm{~g}\right.$ de $\mathrm{LC}$ e $20 \mathrm{~g}$ de diluente); $\mathrm{T}_{50}(50 \mathrm{~g}$ de LC e $50 \mathrm{~g}$ de diluente); $\mathrm{T}_{20}$ ( $20 \mathrm{~g}$ de LC e $80 \mathrm{~g}$ de diluente); diluente (solução de $20 \mathrm{~g}$ de sacarose em $100 \mathrm{~g}$ de leite). X: não houve presença do parâmetro analisado. 
lactose em água e neste trabalho, a matriz é mais complexa quando comparado com a água. Os valores encontrados na literatura para a temperatura de fusão dos cristais de lactose nas amostras $T_{20}$ e diluente são inferiores a temperatura inicial da análise que foi de $25{ }^{\circ} \mathrm{C}$, ou seja, nesta temperatura não seria possível encontrar lactose cristalizada, fato este comprovado experimentalmente.

O ambiente químico das amostras pode ser analisado a partir dos espectros mostrados na Figura 3.

Os espectros Raman são complementares às análises microscópicas realizadas. A partir da interpretação dos espectros pode se concluir que há diferença entre eles, sendo mais evidentes na região de $2900 \mathrm{~cm}^{-1}$, atribuída ao estiramento da ligação $\mathrm{C}-\mathrm{H}$, na região de $1100 \mathrm{~cm}^{-1}$ refere-se ao estiramento C-O e na região de $850 \mathrm{~cm}^{-1}$ atribuída ao modo vibracional da ligação glicosídica dos carboidratos presentes. Com o aumento da diluição há um aumento da quantidade de sacarose no sistema, fazendo com que os espectros fiquem menos definidos, pois os cristais de lactose estão solubilizados no meio (ALMEIDA, 2011).

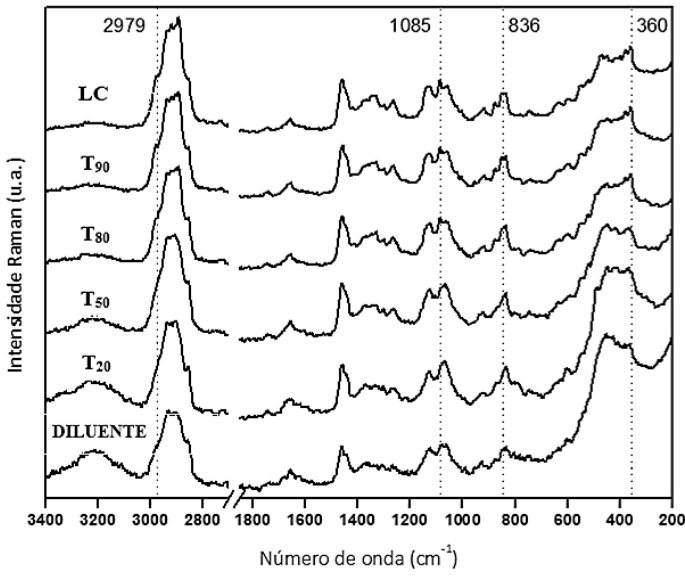

Figura 3 - Espectros Raman das amostras analisadas: $\mathrm{LC}$ (leite condensado puro); $\mathrm{T}_{90}(90 \mathrm{~g}$ de $\mathrm{LC}$ e $10 \mathrm{~g}$ de diluente); $\mathrm{T}_{80}(80 \mathrm{~g}$ de $\mathrm{LC}$ e $20 \mathrm{~g}$ de diluente); $\mathrm{T}_{50}(50 \mathrm{~g}$ de $\mathrm{LC}$ e $50 \mathrm{~g}$ de diluente); $\mathrm{T}_{20}$ (20 g de LC e $80 \mathrm{~g}$ de diluente); DILUENTE (solução de $20 \mathrm{~g}$ de sacarose em 100 $\mathrm{g}$ de leite pasteurizado)

Tabela 4 - Dados da concentração de lactose em cada amostra e temperaturas de fusão dos cristais obtidas experimentalmente por microscopia e temperaturas calculadas de solubilidade inicial da forma $\alpha$-lactose previstas a partir dos trabalhos de Whittier (1944) e Schuck (2018), para soluções de lactose em água nas concentrações correspondentes

\begin{tabular}{cccc}
\hline Amostra* & $\begin{array}{c}\text { Lactose** } \\
(\mathrm{g} / 100 \mathrm{~g} \text { de água })\end{array}$ & $\begin{array}{c}\text { Temperatura } \\
\text { de fusão por } \\
\text { microscopia }\left({ }^{\circ} \mathrm{C}\right)\end{array}$ & $\begin{array}{c}\text { Temperatura calculada da } \\
\text { solubilidade inicial da forma } \\
\alpha \text {-lactose por Whittier; Schuck }\left({ }^{\circ} \mathrm{C}\right)\end{array}$ \\
\hline LC & 40,60 & $83,3 \pm 3,0$ & 80,3 \\
T90 & 32,34 & $65,0 \pm 5,0$ & 71,8 \\
T80 & 26,23 & $56,7 \pm 3,0$ & 64,1 \\
T50 & 14,73 & $41,7 \pm 3,0$ & 42,8 \\
T20 & 8,25 & $\mathrm{X}$ & 21,3 \\
Diluente & 5,31 & $\mathrm{X}$ & 5,0 \\
\hline
\end{tabular}

* LC (leite condensado); $\mathrm{T}_{90}\left(90 \mathrm{~g}\right.$ de LC e $10 \mathrm{~g}$ de diluente); $\mathrm{T}_{80}\left(80 \mathrm{~g}\right.$ de $\mathrm{LC}$ e $20 \mathrm{~g}$ de diluente); $\mathrm{T}_{50}(50 \mathrm{~g}$ de $\mathrm{LC}$ e 50 $\mathrm{g}$ de diluente); $\mathrm{T}_{20}(20 \mathrm{~g}$ de LC e $80 \mathrm{~g}$ de diluente); diluente (solução de $20 \mathrm{~g}$ de sacarose em $100 \mathrm{~g}$ de leite). X: não houve presença do parâmetro analisado.** valores calculados utilizando as análises de composição físico-químicas. 


\section{CONCLUSÃO}

A partir dos dados obtidos experimentalmente, pôde ser encontrada a temperatura de fusão dos cristais de lactose presentes no leite condensado, como também a proporção ajustada para que se possa realizar a etapa de reprocessamento com eficiência. Logo, para a correção da arenosidade foram obtidas duas alternativas. A primeira consiste no aquecimento do produto final acima de $83,3{ }^{\circ} \mathrm{C}$ e a segunda seria diluir o leite condensado na quantidade de $20 \mathrm{~g}$ do mesmo para $80 \mathrm{~g}$ da solução de diluente (20 g de sacarose em $100 \mathrm{~g}$ de leite pasteurizado). Portanto, a temperatura de fusão e a proporção de leite condensado em diluente encontrada neste trabalho pode ser utilizada na indústria de forma a reduzir possíveis prejuízos devido a falha de processamento do leite condensado.

\section{AGRADECIMENTOS}

Os autores agradecem à CAPES (001) e ao CNPq (313088/2017-9; 305513/2018$4 ; 315337 / 2018-4)$ pelo apoio financeiro à realização da pesquisa.

\section{REFERÊNCIAS}

ALMEIDA, M. R. Avaliação da qualidade e variedade de leite em pó e leite condensado por espectroscopia Raman e análise multivariada. 95 f. 2011. Dissertação (Mestrado em Química) - Universidade Federal de Juiz de Fora, Juiz de Fora, 2011.

BRASIL. Ministério da Agricultura, Pecuária e Abastecimento. Secretaria de Defesa Agropecuária. Instrução Normativa $n^{\circ}$ 68, de 12 de dezembro de 2006. Oficializa os Métodos Analíticos Oficiais Físico-Químicos, para Controle de Leite e Produtos Lácteos. Diário Oficial da União: seção 1, Brasília, DF, n. 239, p. 8, 14 dez. 2006.
BRASIL. Ministério da Agricultura, Pecuária e Abastecimento. Instrução Normativa ${ }^{\circ} 47$, de 26 de outubro de 2018. Regulamento técnico de Identidade e Qualidade de Leite Condensado. Diário Oficial da União: seção 1, Brasília, DF, n.213, p. 6, 06 nov. $2018 \mathrm{a}$.

BRASIL. Ministério da Agricultura, Pecuária e Abastecimento. Instrução Normativa $n^{\circ} 76$, de 26 de novembro de 2018. Regulamentos Técnicos que fixam a identidade e as características de qualidade que devem apresentar o leite cru refrigerado, o leite pasteurizado e o leite pasteurizado tipo A. Diário Oficial da União: seção 1, Brasília, DF, n. 230 , p. 9,30 nov. 2018 b.

CODEX ALIMENTARIUS Padrões 2821971/revisão 1999 e 252-2006. Disponível em:http://www.codexalimentarius.net/search/ advancedsearch.do;jsessionid $=$ CCA0FAD3D 8F450945B5FA78DAC548667. Acesso em: 11 nov. 2018.

CUNNIFF, P. A. (ed.). Official methods of analysis of AOAC International. 16th ed. 3th rev. Gaithersburg: AOAC International, 1997.

HOUGH, G.; MARTINEZ, E.; CONTARINI, A. Sensory and objective measurement of sandiness in Dulce de leche, a typical argentine dairy product. Journal of Dairy Science, v. 73, n. 3, p. 604-611, 1990.

IDF - INTERNATIONAL DAIRY FEDERATION. Determination of milk proteins. Standard 20B:1993. Brussels: IDF, 1993. $6 \mathrm{p}$.

IDF - INTERNATIONAL DAIRY FEDERATION. Evaporated milk and sweetened condensed milk: Determination of total solids content. Standard 15B: 1988. Brussels: IDF, 1988. 2 p. 
IDF - INTERNATIONAL DAIRY FEDERATION. Milk: Determination of fat content. Standard 226:2008. Brussels: IDF, 2008. 12 p.

NICKERSON, T. A. Lactose. In: WEBB, B. H.; JOHNSON, A. H.; ALFORD, J. A. (ed.) Fundamentals of Dairy Chemistry. Westport: AVI Publishing, 1974. v. 1, p. 273-324.

NICKERSON, T. A.; MOORE, E. E. Solubility interrelations of lactose and sucrose. Journal of Food Science, v. 37, n. 1, p. 60-61, 1972.

PEREIRA, J. P. F. et al. Water versus lactose solution as a dispersion medium for particle analysis in sweetened condensed milk by laser diffraction. Química Nova, v.42, n.8, p.928-931, 2019. DOI: 10.21577/01004042.20170406 .

PERRONE, I. T. et al. Doce de Leite: Química e Tecnologia. 1. ed. São Paulo: CapLab, v. 1, 2019. 205 p.

RENHE, I. R. T. et al. Characterization of physicochemical composition, microbiology, sensory evaluation and microscopical attributes of sweetened condensed milk. Food Science and Technology, v. 38, n. 2, p. 293-298, 2018. DOI: 10.1590/1678-457x.34416.

RENHE, I. R. T.; PERRONE, I. T.; SILVA, P. H. F. Leite Condensado: Identidade, qualidade e tecnologia. Juiz de Fora: Templo, 2011. 232 p.

SCHUCK, P. Material Didático do Curso: Concentration, Crystallization, Spray Drying e Rehydration. Viçosa, 2018.

SCHUMACHER, A. B. Potencial de utilização de análises de imagens e análises térmicas na cristalização de açúcares em leite condensado. 84 f. 2015 . Tese (Doutorado em Engenharia Química) Universidade Federal do Rio Grande do Sul, Porto Alegre, 2015.

SILVA, F. L.et al. Production of dulce de leche: The effect of starch addition. LWT Food Science and Technology, v. 62, n. 1 , p. $417-423,2015$. DOI: $10.1016 / \mathrm{j}$. lwt.2014.10.062.

SIMEÃO, M. et al. Lactose crystallization in concentrated whey: the influence of vat type. International Journal of Dairy Technology, v. 71, n. 2, p. 478-483, 2018. DOI: $10.1111 / 1471-0307.12455$

STEPHANI, R. et al. Problemas na fabricação de produtos concentrados e desidratados. Informe Agropecuário, v. 38, n. 299, 2017.

WHITTIER, E. O. Lactose and its utilization: A review. Journal of Dairy Science, v. 27, n. 7, p. 505-528, 1944. 